\title{
REAVALIAÇÃO DA FLORA DA FORMAÇÃO POTI: DIPLOTHMEMA GOTHANICA (DOLIANITI) IANNUZZI
}

\author{
ROBERTO IANNUZZI \\ Departamento de Paleontologia e Estratigrafia, Instituto de Geociências, UFRGS, Av. Bento Gonçalves, 9500, \\ Porto Alegre, RS, 91.509-900, Brasil.roberto.iannuzzi@ufrgs.br \\ HERMANN W. PFEFFERKORN \\ Department of Earth and Environmental Sciences, University of Pennsylvania, Philadelphia, \\ PA 19104-6316, USA.hpfeffer@sas.upenn.edu \\ OSCAR RÖSLER \\ Centro Paleontológico, Universidade do Contestado, Cx. P. 111, Mafra, SC, 89.300-000, Brasil.orosler@terra.com.br
}

\begin{abstract}
RESUMO - Neste trabalho examina-se a forma originalmente descrita como Adiantites gothanica Dolianiti, recombinada recentemente pelo autor sênior em Diplothmema gothanica (Dolianiti) Iannuzzi. Em adendo a esta combinação, fornece-se aqui uma descrição mais detalhada desta espécie e uma emenda a sua diagnose original baseada na análise do material tipo e de novos espécimes colecionados por dois dos autores (RI e OR). Além disso, Sphenopteris oliveiranus (Dolianiti) Rigby e S. santosi (Dolianiti) Rigby são consideradas sinonímias de D. gothanica, sendo interpretadas como polimorfias deste tipo de fronde. Por último, a arquitetura da fronde é reconstruída e uma série morfogenética das pínulas é fornecida para este táxon. A distribuição paleobiogeográfica e o biocron de $D$. gothanica são brevemente analisados.
\end{abstract}

Palavras-chave: Pteridophylla, Diplothmema, Carbonífero Inferior, bacia do Parnaíba, Formação Poti, nordeste do Brasil.

ABSTRACT - REVALUATION OF THE FLORA FROM POTI FORMATION: DIPLOTHMEMA GOTHANICA (DOLIANITI) IANNUZZI. This paper is based on the study of Adiantites gothanica Dolianiti, a species recently re-assigned by the senior author as Diplothmema gothanica (Dolianiti) Iannuzzi. In addition to this previous combination, an extended description is furnished but and an emended diagnosis is proposed for D. gothanica based on examination of the type material as well as new material collected by two of the authors (RI and OR). The revision of type collection shows that Sphenopteris oliveiranus (Dolianiti) Rigby and $S$. santosi (Dolianiti) Rigby are synonyms of D. gothanica, being interpreted as polymorphic fragments from this type of frond. Finally, the frond architecture is reconstructed and a morphogenetic series of pinnules presented for this species. The paleobiogeographic distribution and biocron of $D$. gothanica are briefly analyzed.

Key words: Pteridophylla, Diplothmema, Lower Carboniferous, Parnaíba Basin, Poti Formation, northeastern Brazil.

\section{INTRODUÇÃO}

A flora da Formação Poti foi inicialmente reportada por Oliveira $(1934,1935)$ que analisou os espécimes obtidos de um testemunho de sondagem realizado nos arredores de Teresina, no Piauí (poço n ${ }^{\circ} .125$ de Paiva \& Miranda, 1937). Em 1954, Dolianiti realizou um detalhado estudo da flora, tendo para isso re-examinado o material de Oliveira e analisado novos espécimes coletados por Kegel na localidade "Curral de Pedra" (Kegel, 1952). Em seu artigo, Dolianiti (1954) descreveu e classificou a maior parte dos elementos florísticos conhecidos até o momento. Anos mais tarde, ele descreveu três novos componentes da flora encontrados em outras localidades ao sul do Piauí, Maranhão e Pará (Dolianiti, 1962, 1980). Os trabalhos de Dolianiti representam o mais completo estudo já publicado sobre a flora da Formação Poti. Posteriormente, Iannuzzi (1994) fez uma extensa revisão sobre esta flora, baseando-se na re-análise do material de Dolianiti e em novos espécimes coletados por ele mesmo e por Rösler (Rösler \& Ciguel, 1985) em diferentes localidades do Piauí e Maranhão. O estudo de Iannuzzi, entretanto, permanece inédito, uma vez que se trata de uma dissertação de mestrado. Neste contexto, a presente contribuição tem como objetivo apresentar parte dos resultados obtidos por Iannuzzi. Tendo em vista a escassez de dados disponíveis sobre as floras do Carbonífero Inferior da América do Sul (ver síntese em 
Sessarego \& Césari, 1989; Carrizo \& Azcuy, 1997; Iannuzzi et al., 1998; Iannuzzi \& Rösler, 2000), o presente trabalho vem auxiliar no esclarecimento da composição florística da flora da Formação Poti, uma das mais conhecidas e pouco entendidas megafloras do continente.

Neste artigo, examina-se mais especificamente a forma originalmente descrita por Dolianiti (1954) como Adiantites gothanica Dolianiti, recombinada recentemente por Iannuzzi (Iannuzzi \& Pfefferkorn, 2002) em Diplothmema gothanica (Dolianiti) nov. comb. Em adendo à combinação de Iannuzzi, fornece-se aqui uma descrição mais detalhada desta forma e uma emenda à diagnose original. Com isso, a combinação desta espécie no gênero Diplothmema, anteriormente proposta por Césari (1987), é aqui aceita e confirmada, rejeitando-se, contudo, sua sinonimização com a espécie argentina $D$. bodebenderi (Kurtz) Césari, como proposta pela autora (Césari, 1987: 266). Ao final, procura-se elucidar algo sobre a arquitetura da fronde e evolução pinular da espécie em estudo.

\section{GEOLOGIA E MATERIAL}

Todo o material estudado foi coletado em estratos da Formação Poti, na bacia do Parnaíba, nordeste do Brasil (Figura 1). A Formação Poti, originalmente definida por Paiva \& Miranda (1937), é constituída por arenitos finos a médios, esbranquiçados e que apresentam, subordinadamente, níveis de siltitos e argilitos carbonosos intercalados. Estes depósitos são considerados como tendo sido gerados em ambientes flúvio-deltaicos, junto à paleolinha de costa (Schobbenhaus et al., 1984), e representam um trato regressivo dentro do megaciclo "número 1" proposto por Mesner \& Wooldrigde (1964) para as seqüências sedimentares da bacia do Parnaíba. Uma idade eocarbonífera (intervalo Tournaisiano-Viseano) foi anteriormente indicada para os depósitos da Formação Poti a partir dos estudos de seu conteúdo palinológico (Daemon, 1974; Loboziak et al., 1992). Entretanto, uma idade restrita ao Viseano tardio tem sido atribuída com base em recentes e detalhados estudos palinológicos, sintetizados em Melo \& Loboziak (2000). Os restos de vegetais fósseis são mais abundantes na porção superior da unidade.

Os espécimes da coleção tipo são encontrados em amostras retiradas do testemunho descrito por Paiva \& Miranda (1937), proveniente da sondagem $n^{\circ} .125$, realizada na periferia da cidade de Teresina, estado do Piauí (Figura 1). As amostras que contêm os espécimes foram coletadas entre 225 e $226 \mathrm{~m}$ de profundidade, segundo Dolianiti (1954). Os demais espécimes são provenientes de diferentes afloramentos nos estados do Piauí e Maranhão e correspondem ao material adquirido por Rösler (Ciguel \& Rösler, 1985) e Iannuzzi (Iannuzzi, 1994). A maior parte do material coletado em superfície provém da localidade referida por Dolianiti (1954) como "Curral de Pedra". Esta localidade corresponde a afloramentos que ocorrem na área da Fazenda Curral de Pedra, junto à rodovia BR 316, no município de Monsenhor Gil, a 63 km de Teresina, no Piauí (Figura 1). Alguns poucos espécimes foram coletados em um corte de estrada na BR 230, cerca de 23 km de Barão do Grajaú, em direção a São João dos Patos, no Maranhão. O restante do material provém da localidade de Salto de Pedra, no vilarejo de Barra do Lança, distrito de Jerumenha, no sul do Piauí (Figura 1).

Os espécimes provenientes do testemunho da sondagem $\mathrm{n}^{\circ} .125$ e do corte na BR 230 encontram-se preservados em uma matriz pelítico-carbonosa, os da localidade Salto de Pedra em argilito cinza-azulado, enquanto que os da Fazenda Curral de Pedra têm sido encontrados em arenitos finos amarelados a esbranquiçados. Espécimes do testemunho nº. 125, da BR 230 e do Curral de Pedra estão preservados na forma de adpressões. Alguns espécimes desta ultima localidade encontram-se pouco comprimidos, tendo por isso conservado, em parte, sua tridimensionalidade original. Em Salto de Pedra, todos os espécimes correspondem somente a impressões.

Os espécimes tipo (prefixo DGM) estão depositados na coleção paleontológica do Museu de Ciências da Terra do Departamento Nacional de Produção Mineral (DNPM), no Rio de Janeiro. Os demais espécimes (prefixo GP/3T) encontram-se depositados na coleção de paleobotânica do Departamento de Geologia Geral e Econômica do Instituto de Geociências da Universidade de São Paulo (DGG-IG-USP).

\section{BREVE HISTÓRICO}

A espécie aqui estudada foi descrita inicialmente por Oliveira (1935) como Sphenopteris furcata Brongniart. Entretanto, o próprio Oliveira reconheceu que existiam diferenças com a espécie de Brongniart, principalmente quanto à forma obtusa dos ápices dos espécimes brasileiros. Posteriormente, Dolianiti (1954) classificou os espécimes de Oliveira em uma nova espécie, Adiantites gothanica, seguindo, em parte, a orientação do renomado paleobotânico alemão Walter Gothan, feita por meio de uma correspondência. Na verdade, Dolianiti enviou a Gothan a contra-parte de um dos espécimes em estudo, a partir da qual Gothan emitiu um parecer (in Dolianiti, 1954:12-15). Gothan opinou que a forma a ser descrita por Dolianiti pertenceria ao gênero Adiantites por possuir pínulas terminais truncadas, inversamente cuneiformes e com várias nervuras. Acrescentou ainda que esta planta estaria relacionada às formas de Adiantites com pínulas ou folíolos estreitos, pouco comuns ao gênero. Por outro lado, Gothan aconselhou a não criar uma nova espécie, dada a estreita semelhança entre a forma brasileira e Adiantites cf. paracasicus (= Sphenopteris paracasica Gothan, 1928), táxon criado por ele para espécimes provenientes da Península de Paracas, no Peru. Aparentemente, Gothan propôs uma sinonímia entre ambas as formas, recombinando-as em Adiantites cf. paracasicus (Dolianiti, 1954:14). Entretanto, Dolianiti justificou a criação da espécie, Adiantites gothanica, pela existência de finas nervuras ou nérvulos secundários que se interconectavam entre as nervuras e as margens dos lobos (Dolianiti, 1954:1415; est. II, fig. 3). Esta característica não foi observada por Gothan, uma vez que não estava presente na contra-parte 


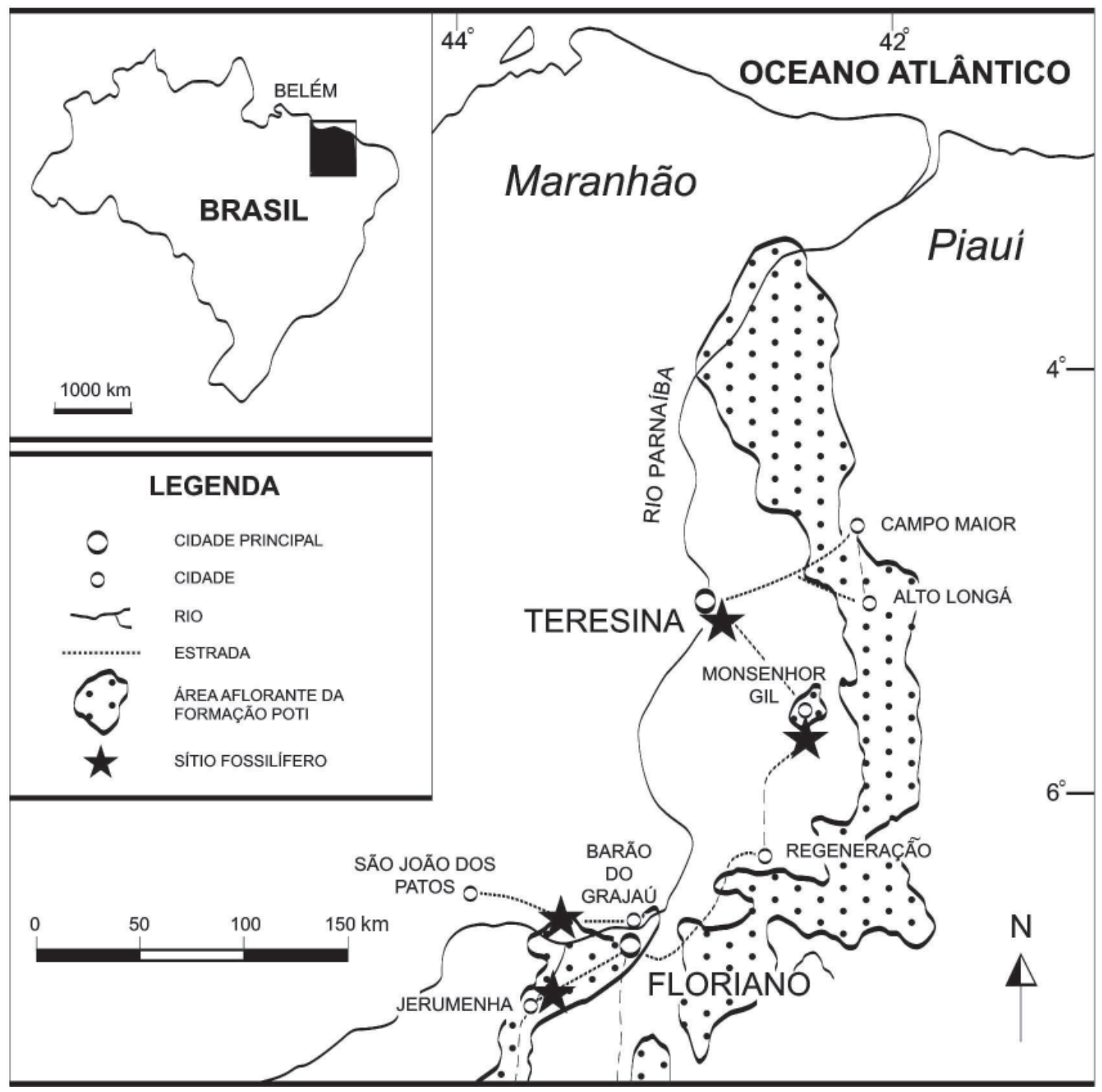

Figura 1. Mapa de localização dos depósitos carboníferos da Formação Poti na bacia do Parnaíba com os sítios (estrelas) dos quais provieram os espécimes de D. gothanica, de norte para sul: sondagem nº. 125, Teresina; Fazenda Curral de Pedra, Monsenhor Gil; BR 230, Barão do Grajaú; Salto de Pedra, Jerumenha.

Figure 1. Location map of Lower Carboniferous deposits of the Poti Formation in Parnaiba Basin with the sites (stars) containing $D$. gothanica specimens, from north to south, as follows: core sampling n 125, Teresina; Curral de Pedra Farm, Monsenhor Gil; BR 230 road, Barão do Grajaú; Salto de Pedra district, Jerumenha.

enviada a ele, segundo Dolianiti (1954:15).

Em 1969, Rigby por sua vez, concordou com as observações de Gothan, tendo considerado que a ausência de nérvulos no material de Paracas estudado por Gothan (1928) poderia ter sido ocasional e que isto não representava uma diferença significativa ou diagnóstica. Assim sendo, Rigby (1969) colocou A. gothanica Dolianiti em sinonímia com $S$. paracasica Gothan, interpretando a descrição de Dolianiti (1954) como uma emenda à descrição original feita por Gothan para o material peruano. Entretanto, seguindo as recomendações que Gothan havia feito a Dolianiti (1954), Rigby recombinou ambas formas em Adiantites paracasica, atribuindo esta nova combinação a Gothan (Rigby, 1969:395396). Sob este mesmo epíteto, Rigby colocou ainda formas afins descritas por Kurtz (1921), Berry (1922), Seward (1922),
Steinmann (1911) e Read (1938) para Argentina e Peru como: Sphenopteris bodenbenderi Kurtz, Palmatoperis furcata (Brongniart) Potonié, Sphenopteris paracasica Gothan e Sphenopteris sp.

Posteriormente, Césari (1987) re-examinou os materiais descritos por Kurtz (1921) como Sphenopteris bodenbenderi e por Frenguelli (1954) como Charnelia dichotoma, estabelecendo para eles uma nova combinação, Diplothmema bodenbenderi (Kurtz) Césari. Neste mesmo trabalho, Césari estendeu esta nova denominação às demais formas anteriormente sinonimizadas por Rigby (1969) como $A$. paracasica. Com isso, o material brasileiro passou a ser incluído em $D$. bodenbenderi (Kurtz) Césari. Recentemente, Iannuzzi (Iannuzzi \& Pfefferkorn, 2002) reconsiderou a espécie de Dolianiti como válida e distinta dos materiais argentino e 
peruano, ao examinar a coleção tipo de Dolianiti e material peruano, além de estudar um novo material proveniente da Formação Poti. Contudo, aceitando os argumentos de Césari (1987) em relação à denominação em nível genérico, Iannuzzi (in Iannuzzi \& Pfefferkorn, 2002) recombinou a A. gothanica de Dolianiti em Diplothmema gothanica (Dolianiti).

\section{PALEONTOLOGIA SISTEMÁTICA}

\author{
Classe PTERIDOSPERMOPSIDA? \\ Ordem PTERIDOSPERMALES? \\ Gênero Diplothmema Stur, 1877 \\ Espécie-tipo. D. schuetzei Stur, 1877
}

\section{Diplothmema gothanica (Dolianiti) Iannuzzi in Iannuzzi \& Pfefferkorn, 2002 emend. \\ (Figuras 2-4; Tabela 1)}

Sphenopteris furcata Brongniart. Oliveira 1935, est. II, III (figs. 1, 7, 9, 11, 12), IV (figs. 6, 8, 10).

Adiantites gothanica Dolianiti 1954, est. I, II.

Adiantites oliveiranus Dolianiti 1954, est. III.

Adiantites santosi Dolianiti 1954, est. IV, V.

Sphenopteris oliveiranus (Dolianiti) Rigby 1969:396, 408.

Sphenopteris santosi (Dolianiti) Rigby 1969:396, 408.

Holótipo. DGM 669, Museu de Ciências da Terra, DNPM, Rio de Janeiro.

Parátipos. DGM 673, 682, 1002, 1009, 1027.

Material estudado. DGM 672, 677, 1006, 1007, 1008; GP/3T $2322,2323,2324,2325,2326,2327,2328,2329,2330,2331,2332$, $2333,2334,2335,2336,2337,2338,2339,2340,2341,2342,2343$, $2344,2345,2346,2347,2348,2349,2350,2351,2352$.

Diagnose original. Pínulas alternas, com contorno geral inversamente cuneiforme, profundamente divididas em 5 a 8 lobos, com terminação arredondada. Os lobos apresentam bordos subparalelos. Das duas nervuras que se apresentam na ráquis, uma única penetra em cada pínula e sofre repetidas divisões, sendo que duas nervuras bem evidentes e de igual intensidade são visíveis em cada divisão lobular. Mas a partir do terço superior de cada lobo, contam-se quatro nervuras que vão até a extremidade do lobo que é arredondado. Pequenos nérvulos partem das nervuras principais e dividindo-se uma e, raramente, duas vezes, atingem o bordo de cada lobo ou se unem à nervura imediata. Esses nérvulos formam um ângulo bem aberto, cerca de $70^{\circ}$, com a nervura principal, e são tão próximos um dos outros que formam uma verdadeira rede. As pínulas são pecioladas (Dolianiti, 1964). Diagnose específica emendada. Frondes tri- a quadripinadas?, de raque principal bifurcada e geniculada. Raques primária, secundária e terciária levemente curvas e sinuosas, portando de forma alterna e em planos ligeiramente diferentes, pinas e pínulas sempre inseridas em ângulos agudos. Pínulas deltóide-alongadas a lanceolados, decorrentes, inseridas por curtas e estreitas bases, profundamente lobadas em segmentos estreitos, de ápices arredondados, inteiros, levemente incisos ou desigualmente bilobulados; pínulas mais basais ao menos pentalobadas, as medianas trilobadas e as apicais simples e indivisas. Venação dicotômica, isotômica, onde uma única veia penetra na base das pínulas e divide-se conforme o número de lobos; duas veias seguem paralelamente por cada divisão lobular e, muitas vezes, estas se dividem distalmente, dando origem a quatro veias; a venação sempre atinge a margem do limbo apenas em sua porção mais distal.

Emended species diagnosis. "Fronds tri-to quadripinnate?; main rachis bifurcate and geniculate. First-, second-and thirdorder rachises slightly curved and sinuous, bearing alternately and in different planes pinnae and pinnules inserted at acute angles. Pinnules cuneiform-elongate to lanceolate, decurrents, inserted to rachis by short leaf-stalks, deeply lobed in narrow segments, tapering to rounded entire, slightly incise or unequally bilobulate apex; basal pinnules of pinnae at least five-lobed, intermediate pinnules tree-lobed and apical ones simple and unlobed. Venation dichotomous, arising from one basal vein that divides according to the number of lobes; two veins per lobe run paralelly to reach the distal portion of leaf margin, many times the veins divides again near to the apex, giving rise to four terminal veins."

Descrição. Impressões e adpressões de fragmentos de frondes bi a tripinadas que alcançam até $78 \mathrm{~mm}$ de comprimento por $52 \mathrm{~mm}$ de largura (Figuras 2, 3). Raque principal (= pecíolo) geniculada, robusta, com finas estrias longitudinais, dicotomizada no ápice, em ângulo de cerca de $80^{\circ}$ e com $12 \mathrm{~mm}$ de largura na base. Raque primária robusta, curta, com $48 \mathrm{~mm}$ de comprimento por $8 \mathrm{~mm}$ de largura, dicotômica no ápice, dando origem a duas raques secundárias de calibre desigual (a menor posicionada externamente) que se dispõem em um ângulo de, aproximadamente, $100^{\circ}$ uma em relação à outra (Figura 3). As raques secundárias internas e externas atingem 6 e 4,7 mm de largura na base, respectivamente, decrescendo gradualmente em direção as porções apicais da fronde até a largura de 1,9 mm (Figura 3).

Nos fragmentos médio-distais da fronde, as raques secundárias são robustas, rígidas, levemente sinuosas, percorridas por um único feixe vascular relativamente largo e suportam, alternadamente, em ângulos agudos, raques de terceira ordem (Figuras 2, 3). Raques terciárias decorrentes, robustas, ligeiramente sinuosas, com largura variando entre 2,2 a 0,8 mm e comprimento máximo de mais $36,6 \mathrm{~mm}$ (Figuras $2,3)$, que dão origem, de modo alterno, a raques quaternárias inseridas em ângulos agudos e em planos ligeiramente distintos ao das raques terciárias. Raques quaternárias rígidas, sinuosas, levemente encurvadas em direção acroscópica, percorridas por um único feixe vascular robusto, com largura entre 0,8 e 1,3 mm na base (em média 1,0 mm), portando pinas de última ordem relativamente curtas, oval alongadas e imparipinadas, que chegam a atingir mais 39,3 $\mathrm{mm}$ de comprimento por 21,9 $\mathrm{mm}$ de largura (Figuras 2,3).

As pínulas são decorrentes, dispostas de forma alterna e inseridas em ângulos agudos e planos ligeiramente distintos ao das raques; apresentam base de inserção constrita e rígida; limbo aparentemente coriáceo, recurvado abaxialmente, reduzido e profundamente lobado; atingem até $9,1 \mathrm{~mm}$ de largura por 15,4 mm de comprimento. As pínulas são 

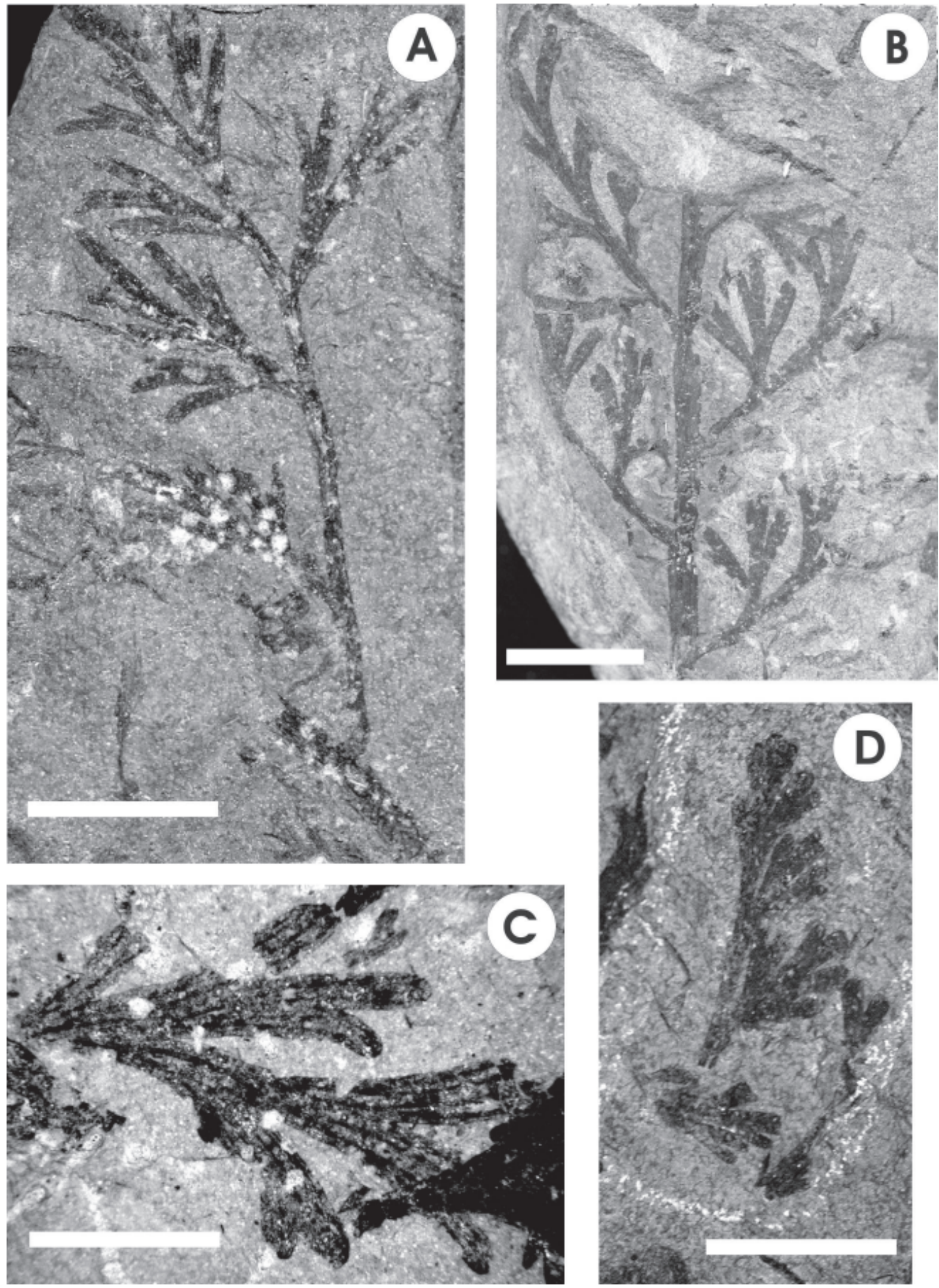

Figura 2. Diplothmema gothanica. A, holótipo, DGM 669, pina de última ordem; B, parátipo, DGM 673, pinas de penúltima e última ordem; C, DGM 1027, pínulas mostrando detalhes da venação; D, holótipo de A. oliveiranus, DGM 677, porção distal de pina de última ordem. Escalas: A, B, D = $1 \mathrm{~cm} ; \mathrm{C}=0,5 \mathrm{~cm}$.

Figure 2. Diplothmema gothanica. A, holotype, DGM 669, ultimate-order pinna; B, paratype, DGM 673, ultimate- and penultimate-order pinnae; C, DGM 1027, pinnules showing details of venation pattern; D, holotype of $A$. oliveiranus, DGM 677 , distal portion of the ultimate-order pinna. Scale bars: $A, B, D=1 \mathrm{~cm} ; C=0.5 \mathrm{~cm}$. 

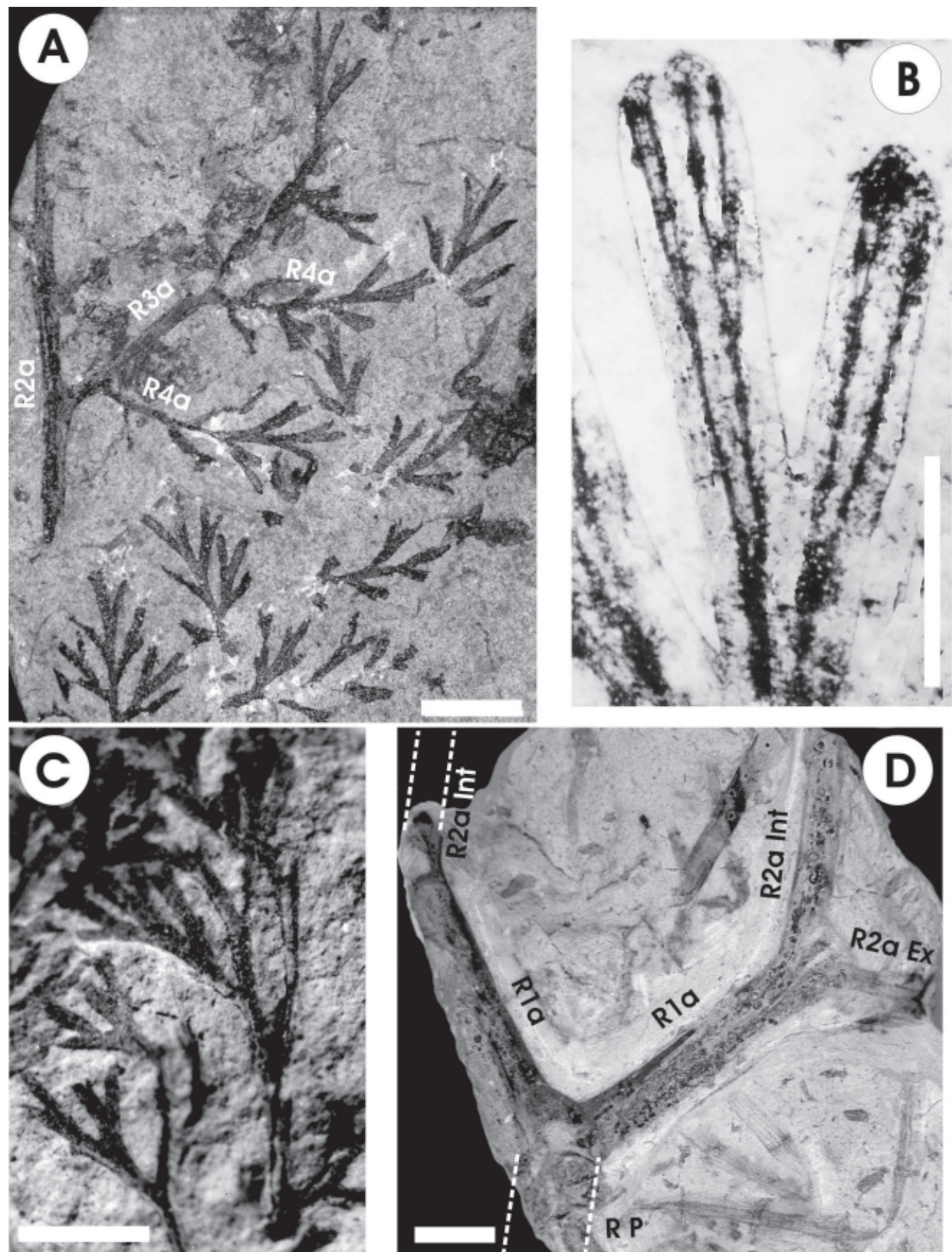

Figura 3. Diplothmema gothanica. A, parátipo, DGM 682, fragmento de fronde com raque secundária(?) e pinas de penúltima e última ordem (Dolianiti, 1954, est. I, fig. 5); B, parátipo de A. santosi, DGM 1009, detalhe da venação das pínulas sem os nérvulos (modificado de Dolianiti, 1954, est. V, fig. 1); C, GP/3T 2323, fragmento de fronde que conserva parte da tridimensionalidade original; D, GP/3T 2333, porção basal de fronde duplamente bifurcada, mostrando raque principal $(R P)$, raque primária $(R 1 a)$ e parte das raques secundárias externa (R 2a ext) e interna (R 2a int). Escalas: A, C, D = $1 \mathrm{~cm} ; C=0,25 \mathrm{~cm}$.

Figure 3. Diplothmema gothanica. A, paratype, DGM 682, fragment of frond bearing secondary rachises(?) and ultimate- and penultimateorder pinnae; B, paratype of $A$. santosi, DGM 1009, detail of the pinnule venation pattern without "nervules" (modified from Dolianiti, 1954 est. V, fig. 1); C, GP/3T 2323, fragment of frond showing the partially preserved original three-dimensional branching system; D, GP/3T 2333, basal portion of bifurcate frond, showing the main rachis (RP), primary rachis (R $1 a)$ and outer (R 2a ext) and inner (R $2 a$ int) secondary rachises. Scale bars: $A, C, D=1 \mathrm{~cm} ; C=0.25 \mathrm{~cm}$. 
A
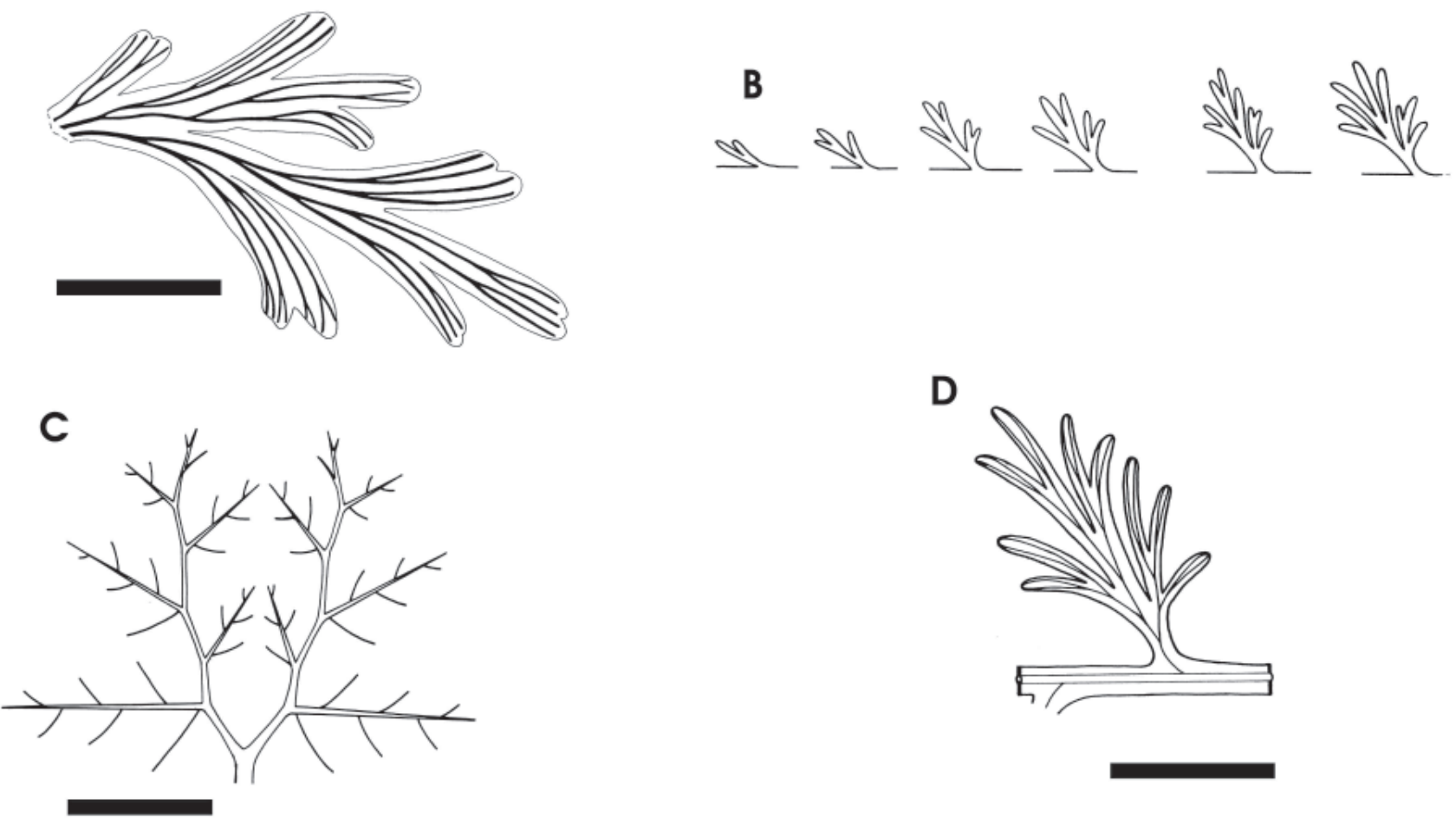

Figura 4. Diplothmema gothanica. A, reconstrução do padrão de venação a partir do espécime DGM 1027; B, desenvolvimento morfogenético das pínulas baseada em espécimes da coleção tipo; $\mathbf{C}$, reconstrução tentativa da arquitetura da fronde proposta a partir de todo material analisado; D, reconstrução de uma pínula madura, notar que um único feixe vascular na raque emite uma única veia que penetra na base da pínula. Escalas: $A, D=0,5 \mathrm{~cm} ; C=5 \mathrm{~cm}$.

Figure 4. D. gothanica. A, reconstruction of the venation pattern based on the specimen DGM 1027; B, morphogenetic development of pinnules observed from specimens of the type collection; $\mathbf{C}$, tentative reconstruction of the frond architecture based on the material studied; $\mathbf{D}$, reconstruction of a mature pinnule, note both the presence of only one relatively wide vascular bundle on the rachis and the unique vein reaching the pinnule leaf stalk. Scale bars: $A, D=0.5 \mathrm{~cm} ; C=5 \mathrm{~cm}$.

polimórficas, variando quanto às dimensões, forma e número de lobos ao longo das pinas (e possivelmente da fronde), sendo as mais basais palmado-alongadas e heptalobadas, as medianas menores, cuneiformes e trilobadas, enquanto que as mais apicais são lanceoladas e indivisas (Figuras 2, 3). Lobos pinulares estreitos, variando em formato de deltóidealongados a lanceolados; de ápices com extremidades arrendodadas e contorno inteiro, levemente inciso ou desigualmente lobulado; atingem, no máximo, $3 \mathrm{~mm}$ de largura por 6,8 mm de comprimento. Lóbulos, quando presentes, são afilados a lanceolados, com largura e comprimento de até 1,3 e 4,2 mm, respectivamente (Figuras 2,3).

A venação é dicotômica, isotômica, derivada de uma única veia basal que se divide tantas vezes quanto forem os números de lobos da pínula, sofrendo uma nova divisão ao penetrar nos lobos, de forma que ocorrem sempre duas veias por segmento pinular (lobo ou lóbulo). Uma vez nos lobos ou lóbulos, as duas veias seguem paralelamente a margem pinular até atingirem o limbo em sua porção mais distal; por vezes, essas veias dividem-se mais uma vez no terço distal, dando origem a quatro veias terminais (Figuras 2, 3).

\section{DISCUSSÃO}

\section{Nérvulos}

Inicialmente, deve-se comentar a possível natureza dos "pequenos nérvulos" descritos (p. 16), ilustrados (est. II, fig. 3; est. IV, fig. 4; est. V) e utilizados (p. 14, 15) por Dolianiti (1954) como uma feição diagnóstica, tendo em vista que os autores tiveram acesso ao material tipo. Não se constatou em quaisquer das pínulas dos espécimes da coleção tipo a presença dos referidos "nérvulos". Por outro lado, os autores tiveram acesso aos fotolitos originais utilizados para elaboração das estampas do artigo de Dolianiti. Baseado na análise deste material fotográfico e nas informações repassadas por D. A. Campos (com. pers.), verificou-se que os tais "nérvulos" foram diretamente retocados nos fotolitos originais com tinta nanquim, a fim de que ficassem em destaque quando da impressão final das ilustrações. Portanto, os "nérvulos" encontram-se demasiadamente destacados nas fotos devido ao efeito causado pela aplicação da tinta. Conclui-se, por conseguinte, que tais "nérvulos" devam corresponder a uma feição preservacional, pouco ressaltada 
Tabela 1. Dados morfométricos (em $\mathrm{mm}$ ) das espécies erigidas por Dolianiti (1954). Em negrito, o novo material de $D$. gothanica coletado pelos autores (GP/3T). Abreviaturas: C, comprimento; L, largura; Lb, largura da base de inserção pinular; N, número de pínulas, lobos e lóbulos analisados.

Table 1. Morphometric data (in $\mathrm{mm}$ ) of the species erected by Dolianiti (1954). In bold, the new material of $D$. gothanica collected by authors (GP/3T). Abbreviations: C, length; L, width; Lb, width of the pinnule leaf stalks; N, number of pinnules, lobes and lobules analyzed.

\begin{tabular}{c|cccc|ccc|ccc} 
& \multicolumn{4}{|c|}{ Pínulas } & \multicolumn{3}{c|}{ Lobos } & \multicolumn{3}{c}{ Lóbulos } \\
\cline { 2 - 12 } & $\mathbf{N}$ & $\mathbf{C}$ & $\mathbf{L}$ & $\mathbf{L b}$ & $\mathbf{N}$ & $\mathbf{C}$ & $\mathbf{L}$ & $\mathbf{N}$ & $\mathbf{C}$ & $\mathbf{L}$ \\
\cline { 2 - 11 } D. gothanica & 32 & $2,4-15,4$ & $0,7-\sim 9,1$ & $0,6-1,4$ & 39 & $2,2-6,8$ & $0,7-2,7$ & 24 & $1,3-\sim 4,2$ & $0,6-1,2$ \\
A. oliveiranus & 10 & $>2,6-\sim 10,4$ & $1,0->6,7$ & $0,6-1,4$ & 17 & $0,6-\sim 7,0$ & $0,6-3,0$ & 12 & $0,8-\sim 4,2$ & $0,8-1,3$ \\
A. santosi & 5 & $>5,2-\sim 8.6$ & $>1,1-\sim 4,6$ & $\sim 0,5-0,9$ & 12 & $1,4-5,1$ & $0,6-1,3$ & 3 & $0,6-0,8$ & $0,5-0,6$ \\
D. gothanica & 24 & $>3,0-9,4$ & $0,5->7,7$ & $0,5-1,0$ & 55 & $>0,6-\sim 4,7$ & $0,5->2,4$ & 47 & $0,6-3,0$ & $0,4-0,8$ \\
\hline
\end{tabular}

na época em que o próprio Dolianiti estudou o material, que foi perdida ao longo desses anos, encontrando-se atualmente ausente nos espécimes analisados. Esta feição pode ter sido criada em algumas pínulas pela degradação gradual de suas películas carbonosas (vê-se nitidamente nas ilustrações de Dolianiti de que esta feição não estava presente em todas as pínulas), pois se constata que o material, em seu estado fresco, deveria ter apresentado películas mais completas, estando preservado preferencialmente na forma de compressões. No seu estado atual, praticamente todas as películas carbonosas foram decompostas, restando alguma matéria orgânica acumulada junto às veias. Neste sentido, descarta-se aqui a presença de "nérvulos" como uma característica diagnóstica da espécie de Dolianiti.

\section{Adiantites oliveiranus e $A$. santosi}

Ao contrário de Diplothmema gothanica, as demais espécies originalmente descritas como Adiantites presentes na flora da Formação Poti, A. oliveiranus e A. santosi, foram erigidas por Dolianiti (1954) com base em material escasso e extremamente fragmentário. Este fato, aliado aos dados levantados pelo autor sênior (RI) ao examinar o material de Dolianiti, revelaram a fragilidade dos argumentos utilizados por aquele autor quando da criação das espécies citadas. Apesar de Rigby (1969) ter recombinado A. oliveiranus e $A$. santosi no gênero Sphenopteris, procurando com isso estabelecer uma firme diferenciação taxonômica entre essas espécies e $D$. gothanica, poder-se-á verificar a seguir o quão próximos encontram-se essas formas de $D$. gothanica. Neste sentido, os autores discordam da opinião de Rigby e consideram os espécimes classificados em Sphenopteris afins aos incluídos em Diplothmema, conforme argumentação abaixo.

Segundo Dolianiti (1954:16-17), A. oliveiranus diferenciase por não apresentar lobos das pínulas tão profundamente incisos ou nérvulos junto às veias principais, além de possuir pínulas quase sésseis e com lobos um pouco mais largos. Nérvulos, como foi visto anteriormente, não devem ser considerados características diagnósticas. Diferentemente do que afirma Dolianiti (1954, p. 16), as pínulas da referida espécie não são "sésseis" no sentido atribuído por ele, mas elas se conectam à raque através de uma curta base de inserção (denominada por ele como "pecíolo"), semelhante a encontrada nas outras duas espécies co-ocorrentes. Uma análise detalhada revelou apenas que a maioria das pínulas parece apresentar uma base de inserção levemente mais larga do que a das demais espécies (Tabela 1). Em relação aos lobos pouco profundos e mais largos, pode-se dizer que estas duas feições encontram-se inter-relacionadas. $\mathrm{O}$ exame dos espécimes atribuídos à A. oliveiranus mostrou que os lobos curtos são resultado de um menor desenvolvimento de suas pínulas como um todo quando comparadas as de $D$. gothanica e A. santosi. Ou seja, não só os lobos, mas as pínulas dos espécimes dessa espécie apresentam-se encurtadas, dando origem a pínulas, em geral, mais curtas (Tabela 1). Este encurtamento das pínulas e lobos dá a falsa impressão de que os lobos de A. oliveiranus são, por sua vez, mais largos do que os das outras espécies erigidas por Dolianiti. Esses lobos porém, demonstram larguras equivalentes às encontradas nos lobos das outras espécies, conforme o presente estudo (Tabela 1).

Neste sentido, verifica-se que há menos características diagnósticas que diferenciem A. oliveiranus, do que previamente havia proposto Dolianiti (1954). Por outro lado, a ocorrência de pínulas e lobos mais curtos, única feição específica validada aqui, não é constatada em todos os espécimes atribuídos a $A$. oliveiranus. O espécime encontrado na amostra DGM 1009 possui, por exemplo, pínulas mais desenvolvidas, com lobos mais compridos, que apresentam dimensões intermediárias entre as típicas de $A$. oliveiranus e as comumente encontradas em $D$. gothanica. Com base nisto, pode-se também questionar a validade desta característica como diagnóstica para os espécimes selecionados por Dolianiti.

Na verdade, Dolianiti (1954) utilizou apenas quatro espécimes para nortear a criação de $A$. oliveiranus, os quais representam somente fragmentos de porções terminais das pinas (1954, est. III). Este procedimento limitou a possibilidade de comparações mais consistentes com espécies definidas a partir de um material mais completo, tal como $D$. gothanica Além disso, sabe-se que é extremamente temerária a criação de novas morfoespécies com base em material escasso e fragmentário, principalmente, no caso de órgãos foliares do tipo fronde, que podem apresentar alto grau de polimorfia ao longo de suas distintas partes. Por outro lado, os espécimes de A. oliveiranus apresentam muito mais feições semelhantes 
aos de $D$. gothanica do que diferenças, como já salientado por Dolianiti (1954), incluindo o padrão morfogenético de desenvolvimento pinular, aferido neste trabalho (Figura 4B). Portanto, uma alternativa seria a de se considerar $A$. oliveiranus como uma variedade de $D$. gothanica $(=D$. gothanica var. minor, p. ex.). No entanto, tendo em vista o exposto, é provável que $A$. oliveiranus corresponda, na realidade, a porções terminais de distintas partes das frondes de $D$. gothanica, que não apresenta espécimes com frondes totalmente completas preservadas até o momento. Portanto, seria mais prudente considerar A. oliveiranus como sinonímia de $D$. gothanica do que manter como válida uma espécie mal estabelecida, deixando-se assim de inflacionar a listagem taxonômica da flora da Formação Poti.

Em relação a A. santosi, Dolianiti (1954) afirmou que esta espécie difere das demais por apresentar pínulas longamente "pecioladas" e de lobos delgados, cujo limbo apresenta numerosos nérvulos que partem das nervuras principais em direção à margem. O caráter preservacional dos nérvulos já foi discutido aqui e estes, por sua vez, não foram constatados no material tipo examinado pelos autores. Quanto às pínulas, uma análise morfométrica dos espécimes de $A$. santosi mostrou que, apesar de suas pínulas apresentarem um ligeiro adelgaçamento nas bases de inserção e nos lobos, em relação à média observada para os espécimes de $D$. gothanica, elas, comparativamente, equiparam-se as medidas obtidas para as pínulas mais estreitas assinaladas em espécimes de $D$. gothanica (Tabela 1). Cabe ressaltar que os espécimes de $A$. santosi, incluindo o holótipo encontrado na amostra DGM 1009 (não em DGM 1008, como citado por Dolianiti, 1954: 20), apresentam os contornos de várias de suas pínulas muito mal preservados, o que, por um lado, inviabiliza a correta delimitação das margens pinulares e, por outro, dá a falsa impressão de que os limbos são mais estreitos ou delgados, principalmente, em suas porções mais proximais. Esta feição preservacional pode ser notada inclusive nas ilustrações do trabalho de Dolianiti (1954, est. IV, fig. 2). Portanto, não há, aparentemente, nenhuma característica diagnóstica que justifique a manutenção destes espécimes em um táxon à parte, tendo em vista a extrema semelhança existente entre estes e aqueles incluídos em $D$. gothanica, como já destacada pelo próprio Dolianiti. Recomenda-se, assim, a transferência dos espécimes de $A$. santosi para $D$. gothanica, evitando-se com isso atribuir distintos nomes para restos tão fragmentários que apresentam apenas pequenas diferenças morfométricas as quais podem ser facilmente aceitas como resultado de uma variação intra-específica.

Do mesmo modo, se aceita os materiais coletados por Rösler (Rösler \& Ciguel, 1985) e por Iannuzzi (Iannuzzi, 1994) como também pertencentes à espécie $D$. gothanica, a despeito das pequenas variações morfométricas apresentadas por alguns de seus espécimes (Tabela 1). Ambos os materiais mostram claramente que a partição em três morfoespécies destes restos de frondes do tipo Diplothmema, como estabelecida por Dolianiti (1954), não é consistente. Os novos espécimes coletados apresentam variações morfométricas de suas pínulas, lobos ou lóbulos, que se sobrepõem às verificadas para os espécimes de cada espécie de Dolianiti, de tal forma que muitos deles não poderiam ser incluídos com precisão em quaisquer das espécies propostas por aquele autor (Tabela 1).

Frondes do tipo Diplothmema, semelhantes às estudadas aqui, são reconhecidamente polimórficas (White, 1943). Isto ocasionou, no passado, grandes problemas taxonômicos, uma vez que era um procedimento extremamente comum aos paleobotânicos identificarem ou mesmo a erigirem uma nova espécie a partir de material fragmentário, como o fez Dolianiti no caso das espécies A. oliveiranus e A. santosi. Entretanto, ficou demonstrado, com o passar do tempo, que mesmo frondes pertencentes a morfogêneros paleozóicos tão distintos como Adiantites, Rhacopteris, Triphyllopteris e Diplothmema, podem apresentar algumas de suas porções praticamente idênticas entre si, inviabilizando, assim, o seu reconhecimento com base apenas em pequenos fragmentos. Portanto, não se recomenda o estabelecimento de entidades taxônomicas baseadas em material fragmentário (White, 1943). Pelo exposto, propõe-se a sinonímia de $A$. oliveiranus e $A$. santosi a $D$. gothanica.

\section{Comparações}

A inclusão da forma de Dolianiti no gênero Diplothmema Stur (1877) promovida por Césari (1987) foi plenamente aceita por Iannuzzi quando do estabelecimento da nova combinação deste material em D. gothanica (Iannuzzi \& Pfefferkorn, 2002). Realmente, dentre os morfogêneros do Paleozóico Superior, Diplothmema é o que apresenta características diagnósticas e espécies mais afins à forma descrita aqui e à espécie de Kurtz, Sphenopteris bodenbenderi, recombinada em Diplothmema por Césari (1987). Deste modo, aceitam-se os argumentos de Césari (1987:265) para justificar recombinação destas formas em Diplothmema. No entanto, cabe salientar que as formas sul-americanas, i. é $D$. gothanica e $D$. bodenbenderi, diferenciam-se por apresentarem, no mínimo, duas veias por segmento terminal (Iannuzzi \& Pfefferkorn, 2002). As demais espécies atribuídas a Diplothmema na Euroamérica possuem apenas uma veia nos segmentos distais das pínulas (Boureau \& Doubinger, 1975). No entanto, a diagnose original do gênero Diplothmema (Stur, 1877, ver Apêndice) não faz menção quanto a esta feição em particular, sugerindo que o padrão de venação não foi inicialmente identificado como uma característica diagnóstica. A opção por incluir apenas formas com segmentos terminais uninervados neste morfogênero, parece ter sido estabelecida pela grande maioria dos paleobotânicos europeus e norteamericanos que posteriormente trataram de classificar e identificar as formas afins ao táxon de Stur, exceto por White (1943) que erigiu algumas espécies norte-americanas de Diplothmema com pínulas de limbo amplo e multinervado.

Talvez, a razão pela qual se tenha optado pela inclusão de apenas formas uninervadas em Diplothmema resida no fato de que a definição e validade deste morfogênero têm sido muito discutidas e questionadas por vários paleobotânicos (White, 1943; Kidston, 1923; Arnold, 1947; White, 1943; Stopa, 1957; Seward, 1962; Alvarez-Ramis et al., 1979), pois 
faltam feições diagnósticas exclusivas do gênero. A caracterização de Diplothmema confunde-se, por exemplo, com as de Palmatopteris e Mariopteris. Na realidade, estes morfogêneros constituem, ao que parece, um "complexo" de formas com feições morfológicas similares que passam gradualmente de um tipo ao outro, o que dificulta a delimitação precisa de cada táxon. Neste sentido, estes táxons necessitam de urgente revisão. Como não se configura no escopo deste trabalho uma análise ou resolução deste problema taxonômico, se aceita por hora a manutenção das espécies sul-americanas em Diplothmema.

Em nível específico, dentre as espécies conhecidas de Diplothmema, a espécie argentina $D$. bodenbenderi é, sem dúvida, a mais semelhante à $D$. gothanica. Iannuzzi (in Iannuzzi \& Pfefferkorn, 2002:577, 584) salientou que $D$. gothanica distingue-se de $D$. bodenbenderi por apresentar pínulas mais estreitas e cuneiformes, nunca palmadas ou orbiculares, nas quais os segmentos terminais são inteiros ou levemente bilobulados, nunca truncados e emarginados. Além disso, destacou ainda como feições diagnósticas de $D$. gothanica a inserção das raques de $3^{\mathrm{a}}$ e $4^{\mathrm{a}}$ ordens e das pínulas sempre em ângulos agudos, nunca retos, e em planos ligeiramente diferentes, dando à fronde uma certa estruturação tridimensional. Com isto, Iannuzzi manteve válida a espécie de Dolianiti, distinguindo-a claramente de $D$. bodenbenderi. Deve-se ressaltar, no entanto, que as porções mais distais de $D$. bodenbenderi assemelham-se as de D. gothanica, podendo ser facilmente confundidas umas com as outras. Por este motivo, não se aconselha tentar o reconhecimento destas duas espécies a partir de materiais muito fragmentados, restritos apenas a suas porções mais apicais (i. é, pínulas e partes distais de pinas de última ordem). Logo, a presença destes táxons em uma determinada flora fóssil deve ser cuidadosamente averiguada e documentada a partir de material mais completo. A figura 4 apresenta a reconstituição do padrão de venação (Figura 4A), a provável reconstituição da arquitetura da fronde (Figura 4C) e a representação ideal de uma pínula madura (Figura 4D) de D. gothanica, a fim de facilitar a identificação e caracterização desta espécie. A mesma figura, mostra o padrão de desenvolvimento morfogenético das pínulas em $D$. gothanica baseado na análise de alguns dos espécimes estudados (Figura 4B).

\section{CONCLUSÕES}

A revisão do material tipo e a análise de novo material coletado por dois dos autores (RI e OR) permitiu ampliar a diagnose original de $D$. gothanica e sinonimizar $A$. oliveiranus e $A$. santosi a esta espécie. Os espécimes que compunham estas duas outras espécies, encontrados em estreita associação com $D$. gothanica, são considerados aqui como diferentes porções da fronde (variações individuais) ou representam variações intra-específicas de $D$. gothanica. Além disso, os autores concordam com a inclusão da forma analisada no morfogênero Diplothmema, como proposta por Césari (1987). Contudo, cabe salientar que as pequenas discrepâncias morfológicas observadas nos espécimes de
D. gothanica e $D$. bodenbenderi em relação aos demais classificados em de Diplothmema (i. é, presença de mais de uma veia por segmento terminal) podem refletir uma diferenciação muito mais acentuada do que parece. Estruturas reprodutivas endêmicas da América do Sul, tais como Kegelidium lamegoi, Occloa cesariana, Obandotheca laminesis e Eonotosperma arrondoi (Dolianiti, 1954; Erwin et al., 1994; Césari, 1997), têm sido encontradas associadas a frondes do tipo Diplothmema em estratos carboníferos da Bacia do Parnaíba, no Brasil, da Península de Paracas, no Peru, e da província de La Rioja, na Argentina, respectivamente. Se confirmada a vinculação entre estas estruturas reprodutivas $\mathrm{e}$ as frondes do tipo Diplothmema, isto poderia justificar a criação, no futuro, de um novo morfogênero para as formas sul-americanas. Todo este conjunto de táxons pode representar, ao que tudo indica, uma nova família de plantas exclusivas do Gondwana ocidental.

Diplothmema gothanica, apesar de ser táxon validado pelo estudo de um bom número de espécimes provenientes de distintas localidades da bacia do Parnaíba, apresenta uma distribuição espacial e temporal extremamente limitadas. Até agora, esta espécie foi constatada, com absoluta certeza, apenas nas associações florísticas da Formação Poti. Ao contrário do que afirmaram Iannuzzi \& Pfefferkorn (2002:584), a ocorrência de D. gothanica na flora de Paracas, no Peru, não pode ser confirmada tendo em vista o material assinalado a esta espécie ser bastante fragmentário. Em termos gondvânicos, indícios da presença desta espécie em floras da África central (Niger) e Índia (Kashmir), como sugerido por Iannuzzi \& Pfefferkorn (2002), podem ser considerados ainda especulativos. Deste modo, D. gothanica tem sua ocorrência limitada à área da Bacia do Parnaíba. Uma explicação plausível para a restrição paleobiogeográfica desta espécie ao nordeste do Brasil poderia ser atribuída aos condicionantes macroclimáticos. De acordo com reconstruções paleoclimáticas de Iannuzzi \& Rösler (2000), a Bacia do Parnaíba encontrar-se-ia em uma zona de clima semiárido durante o Carbonífero, distinta das áreas fitofossilíferas encontradas no Perú, Bolívia ou Argentina, situadas em zonas de climas úmidos. Neste contexto, $D$. gothanica seria uma espécie adaptada às áreas semi-áridas, enquanto que $D$. bodenbenderi poderia ser considerada mais típica das regiões úmidas do continente. Bioestratigraficamente, $D$. gothanica encontra-se restrita aos estratos de idade Eocarbonífera tardia (Viseano tardio), diferentemente de D. bodenbenderi, espécie próxima, que apresenta um amplo biocron, distribuindo-se do Eo ao Neocarbonífero da Argentina e Bolívia (Césari, 1987; Iannuzzi et al., 1999).

\section{AGRADECIMENTOS}

Os autores são muito gratos a Diógenes de Almeida Campos (Museu de Ciência da Terra, DNPM) pelo acesso ao material originalmente descrito por Dolianiti, e pelo seu apoio no presente estudo. Esta pesquisa foi parcialmente financiada pela Fundação de Amparo à Pesquisa do Estado de São Paulo (FAPESP 91/5198-2), Coordenação de Aperfeiçoamento de 
Pessoal de Nível Superior (CAPES, 0461/03-2) e CNPq (PQ 304655/2004-0) na forma de bolsas concedidas (RI). Esta é uma contribuição ao Projeto IGCP 471.

\section{REFERÊNCIAS}

Alvarez-Ramis, C.; Doubinger, J. \& Germer, R. 1979. Die Sphenopteridischen gewachse des Saarkarbons. 2. Teil: Alloiopteris und Palmatoperis. Palaeontographica Abt. B, 170(46):126-150.

Arnold, C.A. 1947. An Introduction to Paleobotany. New York, Mac Graw-Hill, 433 p.

Berry, E. 1922. Contributions to the paleobotany of Perú, Bolivia y Chile. Baltimore, Johns Hopkins University Press, 44 p. (Studies in Geology 4).

Boureau, E. \& Doubinger, J. 1975. Traité de Paléoboatanique. Pteridophylla (1 ${ }^{a}$ partie). Paris, Boureau Ed. Masson et Cie, v. 4 (fasc. 2), $768 \mathrm{p}$.

Carrizo, H.A. \& Azcuy, C.L. 1997. Las fitozonas del Carbonífero temprano de Argentina y la edad de las discordáncias relacionadas: una discussión. Revista Universidade de Guarulhos, Geociências, 2:19-27.

Césari, S.N. 1987. Diplothmema bondenbenderi Kurtz nov. comb. (Pteridospermales?) del Carbonífero da Argentina. Ameghiniana, 24(3/4):263-269.

Césari, S.N. 1997. Eonotosperma arrondoi Césari gen. et sp. nov., a pteridosperm from the Early Carboniferous of Argentina. Ameghiniana, 34(2):169-178.

Daemon, R.F. 1974. Palinomorfos guias do Devoniano Superior e Carbonífero Inferior das Bacias do Amazonas e Parnaíba. Anais da Academia Brasileira de Ciências, 46(3/4):549-807.

Dolianiti, E. 1954. A flora do Carbonifero Inferior de Teresina, Piauí. Departamento Nacional da Produção Mineral, Divisão de Geologia e Mineralogia, 56 p. (Boletim 148).

Dolianiti, E. 1962. Vegetais Fósseis da Bacia Tocantins-Araguaia. Anais da Academia Brasileira de Ciências, 34(4):477-481.

Dolianiti, E. 1980. Rhacopteris na Formação Poti, Estado do Piauí. Anais da Academia Brasileira de Ciências, 52(1):165-169.

Erwin, D.M.; Pfefferkorn, H.W. \& Alleman, V. 1994. Early seed plants in the Southern Hemisphere: I. Associated ovulate and microsporangiate organs from the Carboniferous of Perú. Review of Palaeobotany and Palynology, 80:19-38.

Frenguelli, J. 1954. Plantas devónicas de la Quebrada de la Charnela en la precordillera de San Juan. Notas del Museo de La Plata. Série Paleontolgía, 17(102):359-376.

Gothan, W. 1928. Bermerkubgen sur Alt-Carbonflora von Perú, besonders von Paracas. Neues Jahrbuch für Mineralogie, Geologie und Paläeontologie, Beilagen Band, 59B:292-299.

Iannuzzi, R. 1994. Reavaliação da Flora Carbonifera da Formação Poti, Bacia do Parnaíba. Programa de Pós-Graduação em Geociências, Universidade de São Paulo, Dissertação de Mestrado, 233 p.

Iannuzzi, R. \& Pfefferkorn, H.W. 2002. A pre-glacial, warmtemperate floral belt in Gondwana (Late Visean, Early Carboniferous). Palaios, 17:571-590.

Iannuzzi, R. \& Rösler, O. 2000. Floristic migration in South America during the Carboniferous: phytogeographic and biostratigraphic implications. Palaeogeography, Palaeoclimatology, Palaeoecology, 161:71-94.

Iannuzzi, R.; Díaz-Martínez, E. \& Suárez-Soruco, R. 1999. Los elementos florísticos de la Formación Siripaca (Grupo Ambo, Bolivia) y su contexto biostratigráfico. Pesquisas, 26:21-40.
Iannuzzi, R.; Pfefferkorn, H.W.; Díaz-Martínez, E.; Alleman, V. \& Suárez-Soruco, R. 1998. La flora Eocarbonífera de la Formación Siripaca (Grupo Ambo, Bolivia) y su correlación con la Flora Paracas (Grupo Ambo, Perú). Boletín de la Sociedad Geológica del Perú, 88:39-51.

Kegel, W. 1952. Sobre a Formação Piauí (Carbonífero Superior) no Araguaia. Rio de Janeiro, Departamento Nacional da Produção Mineral, Divisão de Geologia e Mineralogia, 7 p. (Notas Preliminares e Estudos 56).

Kidston, E. 1923. Fossil plants of the Carboniferous rocks of Great Britain. Great Britain Geological Survey, 199-274 p. (Memoir of Paleontology, 2, part 3).

Kurtz, F. 1921. Atlas de plantas fósiles de la República Argentina. Actas de la Academia Nacional de Ciencias de Córdoba, 7:129-153.

Loboziak, S.; Streel, M.; Caputo, M.V. \& Melo, J.H.G. 1992. Middle Devonian to Lower Carboniferous miospore stratigraphy in the Central Parnaíba Basin (Brazil). Annales de la Societé Géologique de Belgique, 115(1):215-226.

Melo, J.H.G. \& Loboziak, S. 2000. Viseán miospore stratigraphy and correlation of the Poti Formation (Parnaíba Basin, northern Brazil). Review of Palaeobotany and Palynology, 112:147-165.

Mesner, J.C. \& Wooldridge, L.C.P. 1964. Maranhão Paleozoic Basin and Cretaceous coastal basins, North Brazil. Bulletin of the American Association of Petroleum Geologists, 48:14751512.

Oliveira, E. 1934. Ocorrência de plantas carboníferas da flora Cosmopolita no Estado do Piauhy. Anais da Academia Brasileira de Ciências, 6(3):113-118.

Oliveira, E. 1935. Sphenopterídeos do Brasil. Anais da Academia Brasileira de Ciências, 7(4):301-312.

Paiva, G. \& Miranda, J. 1937. Carvão mineral do Piauhy. Rio de Janeiro, Departamento Nacional da Produção Mineral, Divisão de Fomento da Produção Mineral, 92 p. (Boletim 20).

Read, C.B. 1938. The age of the Carboniferous strata of the Paracas Pernínsula, Perú. Journal of the Washington Academy of Sciences, 28(9):396-403.

Rigby, J. 1969. A reevaluation of the Pre-Gondwana Carboniferous Flora. Anais da Academia Brasileira de Ciências, 41:393413.

Rösler, O. \& Ciguel, J.H.G. 1985. Plantas fósseis do quilômetro 63 da Rodovia Teresina-Picos, Estado do Piauí (Formação Poti, Carbonífero Inferior?). In: CONGRESSO BRASILEIRO DE PALEONTOLOGIA, 9, 1985. Resumo das Comunicações, Fortaleza, SBP, p. 55.

Schobbenhaus, C.; Campos, D.A.; Derze, G.R. \& Asmus, H.E. (coord.) 1984. Geologia do Brasil. Brasília, Departamento Nacional de Produção Mineral, 501 p.

Sessarego, H. \& Césari, S.N. 1989. An Early Carboniferous Flora from Argentina. Biostratigraphic implications. Review of Palaeobotany and Palynology, 57(3/4):247-264.

Seward, A.C. 1922. On a collection of Carboniferous plants of Perú. Quarterly Journal of the Geological Society of London, 78:278-284.

Seward, A.C. 1962. Fossil plants. New York, Hafner, v. 3, 656 p. Steinmann, G. 1911. Uber die Steinkonlenformation in Sudamerika. Geologie Rundschau, 2(1):50-51.

Stopa, S.Z. 1957. Les feuilles de fougéres (Pteridophylla) du Namurien Superieur et du Westphalien le plus bas dans le bassin houiller de la Haute Silésie. Instytut Geologiczny, 13:1-208.

Stur, D. 1877. Die Culm-Flora der Ostrauer und Waldenburger Schichten. Abhandlungen der Kaiserlich-Königlichen Geologischen Reichsanstalt, 8(2):1-366. 
White, D. 1943. Lower Pennsylvanian species of Mariopteris, Eremopteris, Diplotmema and Aneimites from the Appalachian region. U.S. Geological Survey, 140 p. (Professional Papers, 197-c).

Received January, 2006; accepted March, 2006

Apêndice. Transcrição e tradução da diagnose original do gênero Diplothmema Stur.

Appendix. Transcription and translation of the original diagnosis of genus Diplothmema Stur.

Diplothmema Stur, 1877

Espécie tipo: Diplothmema schuetzei Stur

O gênero Diplothmema é tratado nas páginas 120-148. A espécie $D$. patentissima (Ettingh.) Stur é freqüentemente citada como espécie tipo, mas não o é. Apesar de Stur a mencionar primeiro em seu trabalho, ela é citada como "cf.". Portanto, esta não é válida como tipo, pois de acordo com o Código Internacional de Nomenclatura Botânica (ICBN) nomes ou designações nas quais o autor expressa qualquer dúvida não serão validadas. Deste modo, $D$. schuetzei é a espécie tipo do gênero, uma vez que surge a seguir dentro do trabalho e foi estabelecida por Stur sem dúvidas, tendo sido plenamente descrita e extensivamente figurada.

\section{Diagnose Original}

"Caudex epigaeus, cylindricus, subtilior vel crassus trichomatorum cicatriculis otectus vel longitudinaliter striatulus, ecarinatus vel obsolete aut et evidenter carinatus, ad carinas saepe alatas transverse rugosus; petioli in caudice spiraliter dispositi, distantes, articulati, superne canaliculati inferne linea prominente notati, longitudinaliter striolati medio transverse rugosi vel laeves aut trichomatosi, nudi, apice in duos ramos sub angulo $30-175$ graduum divergentes, strictos vel geniculatos, plus minus elongatos, furcati; folii lamina ambitu non raro formam semilunae dorso petiolatae referens, nude petiolata in duas sectiones plus minusve divergentes, katadrome auctas, interse symmetrice 1-5 pinnatisectas 1. - pinnatifidas secta, lamina sectionum in lacinulas lineares 1. lanceolatas aut et cuneatas plus minus subrotundas, rarius in segmenta pinnatipartita 1. pinnatilobata, divisa; fructificatio folii fructiferi, paginam inferiorem sectionis mediae, integrae, ambitu subrotundae (in folio fertili tantum evolutae?) occupans".

\section{Tradução da Diagnose Original}

Caule aéreo, cilíndrico, liso ou coberto com grossas cicatrizes de pêlos ou finamente estriados longitudinalmente, carenados ou desgastados ou mesmo com nítidas carenas, muitas vezes alas rugosas encontram-se transversalmente aderidas às carenas; pecíolos dispostos espiraladamente no caule, com sulcos acima do ponto de inserção foliar e com saliências proeminentes abaixo, longitudinalmente estriados no meio com rugas transversais ou lisos ou pilosos, nus (= sem pinas ou pínulas), bifurcados no topo em dois ramos que divergem em ângulo de $30^{\circ}-175^{\circ}$, retos ou geniculados, mais ou menos elongados, furcados; lâmina das folhas (= fronde) em sua circunferência de formato normalmente em meia-lua em relação ao dorso do pecíolo, com pecíolos nus divididos mais ou menos em duas seções, sendo o lado catadrômico mais desenvolvido, entremeadas simetricamente por segmentos 1-5 vezes pinados - nas porções pinatífidas (= pínulas e pinas em diferenciação), lâminas divididas em segmentos lineares estreitos (= lobos) - lanceolados ou cuneiformes mais ou menos subarredondados, raramente pinados em segmentos ou pinadamente lobados, divididos; frutificações em folhas férteis, sobre o lado inferior na seção mediana, inteira, em circumferência aproximadamente arredondadas (em folhas férteis mais ou menos desenvolvidas)". 